\title{
MAS Background Correction Method
}

National Cancer Institute

\section{Source}

National Cancer Institute. MAS Background Correction Method. NCI Thesaurus. Code C64237.

This is an implementation of the Affymetrix (2002) MAS algorithm for background correction, which divides each chip into regions, uses a threshold to identify a 'background' level of expression, and subtracts this from both MM and PM probes. 